University of Nebraska - Lincoln

DigitalCommons@University of Nebraska - Lincoln

Faculty Publications, Department of Psychology

Psychology, Department of

May 1977

\title{
Stability of Perceived Tension as a Function of the Menstrual Cycle
}

\author{
Paula Englander-Golden \\ University of Oklahoma
}

Keith A. Willis

Willmar State Hospital, Willmar, Minnesota

Richard A. Dienstbier

University of Nebraska-Lincoln, rdienstbier2@unl.edu

Follow this and additional works at: https://digitalcommons.unl.edu/psychfacpub

Part of the Psychiatry and Psychology Commons

Englander-Golden, Paula; Willis, Keith A.; and Dienstbier, Richard A., "Stability of Perceived Tension as a Function of the Menstrual Cycle" (1977). Faculty Publications, Department of Psychology. 162.

https://digitalcommons.unl.edu/psychfacpub/162

This Article is brought to you for free and open access by the Psychology, Department of at DigitalCommons@University of Nebraska - Lincoln. It has been accepted for inclusion in Faculty Publications, Department of Psychology by an authorized administrator of DigitalCommons@University of Nebraska - Lincoln. 
Published in Journal of Human Stress 3 (June 1977), pp. 14-21. Reprinted with permission of the Helen Dwight Reid Educational Foundation. Published by Heldref Publications, 1319 18th Street, NW, Washington, DC 20036-1802. Copyright (C) 1977.

\title{
Stability of Perceived Tension as a Function of the Menstrual Cycle
}

\author{
Paula Englander-Golden, Ph.D., Keith A. Willis, Ph.D., \\ and Richard A. Dienstbier, Ph.D.
}

\begin{abstract}
The stability of tension judgments among males and females who were either taking or not taking combination low-progestin oral contraceptive pills was investigated. Judgments of interpersonal pressure in videotaped vignettes provided tension measures. The vignettes were presented in two sessions separated by two weeks. Correlations between pressure judgments of .94 for males, .79 for pill females and .40 for non-pill females (all sig <.002) supported the prediction that non-pill females experience the largest fluctuations in tension across the menstrual cycle. A significant number of non-pill females who changed their scores and who changed from midcycle to premenstrual-menstrual phases or vice versa between the two experimental sessions shifted their tension scores in the predicted direction ( $p<$ .04). As predicted, non-pill females scoring in the lower half on premenstrualmenstrual symptomatology were more consistent in pressure judgments than those high on symptomatology, with correlations of .51 (sig <.004) and .24 (NS) respectively. Analysis of pressure judgments as a function of menstrual phase and high-low symptomatology indicated a main effect for symptomatology (sig < .01) with the high group perceiving more pressure. Lack of a main effect for menstrual phase was attributed to large individual variations in tension scores. Results were interpreted as indicating physiological causality underlying the variability in tension across the menstrual cycle. Substituting the Repression-Sensitization dimension for symptomatology yielded no significant results which could lead to a psychological interpretation of such variability.
\end{abstract}

Although the "premenstrual syndrome" has been poorly defined, it is reported by various authors to affect between 25 and 100 percent of women. ${ }^{1}$ In a review of the premenstrual syndrome Parlee ${ }^{2}$ cited several studies which reported an increase in tension, anxiety, depres- sion, irritability, commission of violent crimes, suicides and accidents in the late premenstrual or early menstrual days.

Different studies can be cited to support either a physiological or social etiology for the cyclical variations in moods. For instance, in a

Dr. Englander-Golden is Assistant Professor in the Department of Human Relations at the University of Oklahoma, Norman, Oklahoma. Dr. Willis is Clinical Psychologist at Willmar State Hospital, Willmar, Minnesota. Dr. Dienstbier is Chairman of the Department of Psychology at the University of Nebraska-Lincoln, Lincoln, Nebraska. 
study in which some women took contraceptive pills while others did not, Paige ${ }^{3}$ concluded that social rather than physiological causes mediate anxiety which reaches a peak during the menstruating phase; she suggested that hostility (defined as a combined score on inward, outward, and ambivalently directed hostility), which reaches a peak premenstrually, may be biochemically determined. Supporting a biochemical mediation viewpoint are studies which relate directly the premenstrual decrease in estrogen and progesterone to an increase in monoamine oxidase (MAO) activity. This increase in MAO is in turn related to moods such as tension and depression. ${ }^{4 *}$ For healthy women MAO activity is lowest at the time of ovulation (mid cycle) at which time it is significantly lower than that in males; MAO increases premenstrually to a level similar to males. Menopausal women are higher on MAO activity compared to healthy menstruating women and are reported to be more tense. MAO levels remain relatively stable across the menstrual cycle in women taking combination low-progestin oral contraceptive pills and tend to be relatively stable for males. ${ }^{6,7,3}$

The present study was designed to investigate cyclical variations in tension reported to occur in women. To distinguish cyclical variations associated with the menstrual cycle from the variations due to other causes, a control group of males was used. A second control group was also included which was comprised of women taking oral contraceptive pills of the combination low-progestin type. As indicated above, such contraceptives are reported to keep levels of MAO relatively stable across the menstrual cycle. Tension levels were assessed at two different times separated by an interval of two weeks.

* MAO is measured endometrially or in plasma but it is assumed that such measurements reflect MAO activity in the brain. Broverman et al. ${ }^{5}$ hypothesized that MAO partly controls levels of norepinephrine, a neural transmitter of the central adrenergic nervous system. High levels of MAO result in impaired adrenergic functioning by leading to diminished neural norepinephrine. Psychiatrically depressed patients have significantly higher plasma MAO activity than nondepressed people.
Previous research ${ }^{8}$ had indicated that when female subjects were given information that a stimulus person was either pre- or post-menstrual, they tended to attribute the causes of the described moods differentially. Unfortunately this type of approach gives no suggestion as to whether such attributions occur because the female subjects experienced their own moods differentially across the menstrual cycle, whether they simply assumed that the stimulus person would experience hers differentially, whether they responded to some other aspect of the cultural stereotype, or whether they responded to the demand characteristics of the experiment. Our research was designed specifically to answer the question of whether women in different phases of their menstrual cycles experienced different mood states, even though the topic of menstruation was not salient in the experimental situation. Furthermore, we anticipated that one of the most important results of experiencing menstrual mood changes is to perceive different degrees of threat, pressure, and potential stress in interactions with others. We therefore utilized a projective approach in which subjects viewed a series of short videotaped vignettes of two people interacting over issues. The vignettes were designed to be neutral with respect to interpersonal pressure, so that the perception of pressure would reflect the subject's own state of tension as much as possible. By having the same subjects view equivalent vignettes twice, with a two-week interval, it was possible to study the degree of consistency which the three different types of subjects experienced in interpersonal pressure in the vignettes. It was predicted that the correlations between pressure scores for the two sessions would be highest for men, next highest for women taking oral contraceptive pills, and lowest for women not taking any kind of oral contraceptives.

Additionally, within the subject group of women not taking the pill, we anticipated a great deal of individual variation in consistency of pressure perception across the two sessions, due to differences in degree of experienced cyclical variations in mood across the menstrual cycle. Indeed, the wide disparity of symptomatology which different authors have report- 
ed (see above) suggests such individual differences. Since our approach was based upon the belief that underlying physiological differences would mediate these mood cycle differences, subjects were asked to respond to a symptomatology questionnaire which sought to determine if they experienced premenstrual or menstrual symptoms such as cramps, weight gain, physical tension, and depression. Dividing these subjects into high and low symptomatology groups, it was predicted that the low group would have a higher correlation on perceived pressure scores between the two sessions than would the high group.

Byrne's Repression-Sensitization (RS) Scale ${ }^{9}$ was administered to rule out the competing hypotheses that consistent anxiety or a willingness to admit to socially undesirable attributes could account for the anticipated differences in the stability of pressure judgments. Lazarus, et al. ${ }^{10}$ suggested that the RS scale could be measuring both anxiety and social desirability. Finally, based upon the physiological changes associated with the phases of the menstrual cycle and upon a pilot study conducted near the end of the previous semester, it was predicted that the perception of pressure would be related to the menstrual phase of the non-pill subjects. For the non-pill group it was predicted that the average value of perceived tension would be lowest for women at mid cycle. Furthermore it was hypothesized that non-pill women who change their tension scores when they change from the mid cycle phase to the premenstrual or menstrual phases, in the two week interval separating the two experimental sessions, would shift to higher tension score values. The opposite prediction was made for women who change from the premenstrual or menstrual phases to the mid cycle phase in the two week interval. High levels of estrogen associated with this phase are hypothesized to be the hormonal basis for the general feeling of well-being experienced by mid cycle women. ${ }^{11,12}$ No difference was hypothesized for women at the premenstrual vs. menstrual phase of their cycles. During the premenstrual phase it is the plunging levels of estrogen and progesterone which are thought to underlie the tension and anxiety experienced then, and the relatively low levels of those same hormones are believed to account for similar negative moods during menstruation. ${ }^{11}$

\section{METHOD}

\section{Subjects}

Subjects were introductory psychology students who agreed to participate in this research rather than fulfill their research requirements in other ways. Females were selected at random, that is, by social security number. Males were obtained by "sign up sheet" for experimental participation. Twenty-five males and eighty-four females participated; it was later determined that 18 of the females were taking combination lowprogestin oral contraceptives.

\section{Procedure}

At the beginning of the semester the subjects were asked to fill out Byrne's RS scale. At that time they were also told that they should return for two experimental sessions scheduled later in the semester.

Subjects appeared in the experimental sessions in groups of 6 to 15 with females and males participating separately. A female experimenter was assigned to the female groups and a male to the male groups.

At the first of the two experimental sessions, which occurred in the third week of the semester, the experimenter introduced herself/himself and described the sequence of activities which would follow. The videotape recorder was then turned on. Subjects were typically 10 feet from the nine-inch Sony video monitor.

The videotape explained that subjects would evaluate videotaped vignettes which would later be used in the public school system to study cognitive development in third and fourth graders, particularly the manner in which such children make inferences from observations. Thus the videotapes demonstrated "inference-making from observations," but the videotapes were "supposedly constructed to be free from interpersonal pressures associated with tone of voice, facial expression, or body movement." It was explained that the subjects' task was to 
evaluate the vignettes for signs of such pressure so that only the most neutral vignettes would be used with the children. Finally, it was stated that the 12 vignettes presently ready for viewing did seem to the experimenter to be free from the more obvious possibilities of such pressures. Instructions were then given on scoring for pressure. Subjects were told they would be given time between each vignette to make and record their judgments.

When the cover story was finished, the videotape recorder was turned off. The experimenter then asked if the instructions were clear and said that the reception in the building was somewhat poor so they should simply do their best on the vignettes. Then 12 vignettes were shown.

The vignettes showed two protagonists, either two brothers about 9 to 10 years of age, a mother and one of the boys, or a father and one of the boys. Twelve vignettes were presented such that each subject viewed an equal number of boy-boy, mother-boy, and father-boy vignettes. Protagonists were counterbalanced regarding who made the opening statement, and all vignettes were presented in random order. The following is an example of a vignette:

Two protagonists enter a room.

John: Look, Dad, the shade is open. I think Steve opened it to get some light in the room.

Dad: Yes, John, the shade is open, but I think Steve opened it to look outside.

Announcer: (only his voice is heard) Could John be right when he says that Steve opened the shade to get light in the room? Could John's dad be right when he says Steve opened it to look outside? Could both John and his dad be right? Are none of them right?

Although in the cover story subjects were also asked to judge the announcer for possible pressure, the tension score has been limited solely to judgments regarding the protagonists. The maximum score a subject could get was 24 . This score could be obtained by scoring both protagonists in every vignette as communicating nonverbal interpersonal pressure.
When this part of the experiment was completed, a different set of tasks (performance tests and a mood questionnaire) was given to the subjects for a different investigation. At the end of the session, the subjects were asked to make appointments for the second experimental session exactly two weeks later.

The experiment was repeated two weeks later without the introductory cover story. At the end of the second session, the subjects filled out a questionnaire designed to uncover any suspicions they might have regarding the purpose of the experiment. The questionnaire was a funnel type questionnaire ${ }^{13}$ beginning with general questions and ending with specific questions concerning suspicions about the hypotheses and details of the study.

\section{Determination of Menstrual Phases}

During debriefing it was explained to the female subjects that for an accurate determination of the midcycle and premenstrual phases one needs to count backward from the onset of the following menstruation. Subjects were then asked to inform the experimenter as soon as the onset of their next menstruation occurred. Subjects were also asked to indicate the onset of their last period. This question enabled the identification of subjects who may have been menstruating during the first or second experimental session.

For purposes of data analysis, women were defined as at mid cycle during days 13, 12, 11, $10,9,8$ and 7 prior to menstruation onset.* Premenstrual days were defined as days 5, 4, 3,2 , and 1 prior to menstruation; if subjects

*The rationale for defining days 13 through 7 prior to the onset of menstruation as mid cycle was based on the following considerations. Estrogens rise rapidly and reach a peak just before ovulation. However, within hours after the beginning of the luteinizing hormone (LH) surge, and before the rupture of the follicle, there is a precipitous drop in plasma estrogen. Estrogen begins to rise again during day 13 prior to the onset of menstruation. ${ }^{14}$ In some women the preovulatory drop in estrogen may be associated with high levels of MAO. ${ }^{15}$ Unless pregnancy intervenes, the levels of estrogen and progesterone begin to drop 7-8 days after ovulation. ${ }^{14}$ Since the LH peak occurs at day 15 prior to onset of menstruation and rupture of the follicle occurs 16-24 hours after the beginning of the LH surge, ${ }^{14}$ it was assumed that day 13 would be postovulatory for most women. Day 7 prior to onset of menstruation was included because levels of estrogen and progesterone appear to be still relatively high. ${ }^{14}$ 
began menstruating after participating in the study on day 0 (i.e., the day of the experiment) they were also classified as premenstrual. Subjects were classified as menstruating if they were in days $1,2,3$, or 4 following menstruation onset, or if they participated in the study on day 0 after beginning menstruation.

\section{Determination of Symptomatology and Contraceptive Use}

At the beginning of the debriefing session, after being assured that they had already completed all requirements for their research participation, the female subjects were asked to fill out the symptomatology questionnaire for several phases of the menstrual cycle. The symptoms reported were cramps, headaches, weight gain, physical tension, crying spells, lump-inthe-throat, depression and "tight band" around the head. Each symptom could be judged from very severe (assigned a value of 1) to none (assigned a value of 4). Two intermediate values were also possible. The symptomatology score was composed of the sum of the premenstrual and menstrual scores. The high and low symptomatology groups were formed by a median split on the premenstrual-menstrual symptomatology (PM) scale. Information about use, type, and dosage of contraceptives was obtained at the end of the full debriefing session in which the purpose of the study was explained in detail. Subjects were assured complete anonymity and were again told that giving this information was voluntary but very important to the completion of the study. No subject refused to provide the requested information.

Debriefing for the male subjects included a discussion of theories of biological rhythms and cyclical variations in moods, omitting an extensive discussion of menstrual cycles.

\section{RESULTS}

At the first session 84 females participated. Of these, only 78 participated in the second session where it was ascertained that 23 were taking contraceptive pills. Only 18 were taking combination low progestin type pills. Fifty-five females were not taking any oral contraceptives. However, one was pregnant, one was a foreign student with language difficulties, and one was told about the experiment by a friend; thus, the non-pill female group was reduced to 52. The RS score for one of these subjects could not be found. An attempt was made to determine why the other six females did not return for the second session so that it could be ascertained whether biasing due to absences related to menstruation had occurred. It was found that one had pneumonia, one had the flu and one was a nurse on call in a local hospital; three subjects could not be located.

Of the 25 males who participated in the first session, 22 returned to the second session.

The Pearson correlation coefficients for interpersonal pressure judgments between the two experimental sessions were $.94(\mathrm{p}<.001, \mathrm{~N}=$ 22) for males, $.79(p<.001, N=18)$ for the pill women, and $.40(\mathrm{p}<.002, \mathrm{~N}=52)$ for the nonpill women. Significance of the differences between the correlations was tested using Fisher's $\mathrm{Z}$ transformation technique. ${ }^{16}$

It was predicted that since the pill women would have less extreme mood swings through their menstrual cycle, they would be more consistent in pressure judgments between the two sessions than the non-pill women, but less consistent than men. The correlation for the pill women was significantly different $(\mathrm{p}<.05)$ from those for the other two groups; the correlation for the non-pill women was significantly different from that of men.

When the non-pill female group was divided into those high and those low on symptomatology, a correlation between the two pressure judgments of .51 ( $<<.004, \mathrm{~N}=25)$ was achieved for the low group, and $.24(\mathrm{NS}, \mathrm{N}=27)$ for the high group. Since the symptomatology variable therefore appeared to be an important factor in differentiating between pressure-judgment consistency within the non-pill group, these results supported the interpretation that the differences between the three major subject groups were due to the differing degrees to which menstrual cycling was experienced. A similar division of the non-pill group into high and low sensitizers on Byrne's RS scale found correlations of $.63(\mathrm{p}<.001, \mathrm{~N}=24)$ for the low scorers or repressors, and $.32(\mathrm{p}<.05, \mathrm{~N}=27)$ for the sensitizers across the two experimental sessions. 
Thus, the differences in consistency between the pill women and the non-pill groups are attributable to those non-pill women who report high symptomatology (those correlations differ significantly, $\mathrm{p}<.01$ ); when the alternate division is made of non-pill group, it is the sensitizer group which differs significantly $(p<.05)$ from the pill women. The RS and symptomatology scales have been hypothesized to measure different variables and, indeed, the obtained correlation between the two scales was low, .35 . However, to ascertain that this low correlation was due to measurements of different variables one needs to know that the reliabilities of the two scales are relatively high. Test-retest reliability for the RS scale is $.82 .{ }^{17}$ The reliability for the symptomatology scale is not available. However, this scale has similar questions to those given by Moos ${ }^{1}$ in his Menstrual Distress Questionnaire, Moos ${ }^{1}$ reports that women tend to have consistent symptomatology from one menstrual cycle to another. Thus, perhaps with a certain degree of confidence one can assume that the two scales do not measure the same variable.

Differences in the absolute levels of pressure judgments between women in different points of the menstrual cycle were predicted for the non-pill group; such differences were also expected between those scoring high and low on symptomatology and the RS scale. Contrary to our expectations that midcycle women would perceive less pressure than menstrual or premenstrual women, a $3 \times 2$ factorial analysis of variance (with high or low symptomatology the second variable) indicated no main effect for cycle $(\mathrm{F}=1.60, \mathrm{df}=2,39, \mathrm{NS})$.

To gain further insight into the failure to identify that expected main effect for menstrual phase, the 12 women who were in the two sessions of the study during both their midcycle phase and the premenstrual-menstrual phase were studied closely. Of those 12, 11 changed

\footnotetext{
* Each subject appears only once in the analysis of variance. However, some females were in two different menstrual phases at the two experimental sessions; only one of their scores was used. When a choice had to be made it was always the mid cycle score that was dropped, since midcycle cells had more data than premenstrual or menstrual cells.
}

their pressure judgments between the two sessions, with nine indicating lower scores and two indicating higher scores at mid cycle. A binomial analysis, which considers only the direction of change, indicates the probability of this large a difference to be $p<.04$. However, a t-test for correlated measures between the two sets of tension judgments (means of 6.67 at mid cycle vs 8.00 at premenstrual-menstrual phase, $\mathrm{t}=$ $1.46, \mathrm{p}<.10)$ does not indicate statistical significance. The prediction of increased pressure judgments during the premenstrual-menstrual phase is therefore weakly supported for the women who should have provided the clearest test of that hypothesis.

A main effect for symptomatology appeared with those high in symptomatology receiving an average pressure score of 8.70 compared to 5.86 for those low $(\mathrm{F}=7.83$, df $=1,39$, sig < $.01)$; no interaction was indicated. When pressure was analyzed in a second $3 \times 2$ analysis of variance, using the variables of cycle and RS, no main effects or interaction appeared. Finally, disregarding the issue of menstrual cycle, differences in absolute pressure judgments between the three main subject groups were examined, though no prior hypotheses were tested. No meaningful or significant differences were noted.

\section{DISCUSSION}

By having subjects respond to perceived pressure twice, across a two-week interval, it was our assumption that for most of the women subjects, the two sessions would occur at significantly different points of their menstrual cycle. To the extent that the physiological concomitants of the menstrual cycle dispose the mood changes normally associated with such cycling, it was anticipated that those subjects for whom the menstrual cycling was most variable would respond least consistently on the two occasions, and that the non-pill females would experience least tension at midcycle. These expectations received partial confirmation from the finding that non-pill women were significantly less consistent in their judgments of interpersonal pressure than were pill women, who were in turn less consistent than males. Furthermore, a signifi- 
cant number of non-pill females who changed their pressure scores when they changed menstrual phases during the two-week interval separating the experimental sessions, did so in the predicted direction. Although the lack of significant difference between the two sets of pressure judgments (premenstrual-menstrual versus midcycle) for this group of females weakens our physiological argument, this weakness in the data may be attributed to both the small sample size and large individual variability in tension scores. It is also possible that in real life a strong increase in premenstrual-menstrual tension results from an interaction between physiological factors and stressful events. The present experiment was not presented in a stressful manner and it was performed at the beginning of the semester when real life problems may have been relatively low.

Our hypothesis receives crucial support from the finding that within the group of non-pill women high premenstrual-menstrual symptomatology was related to inconsistency of pressure judgments between the two sessions. Similar differences did not appear when the non-pill women were split into high or low scorers on the RS dimension. Further research is needed to explain the obtained relationship between symptomatology and perceived pressure, with high symptomatology non-pill women perceiving significantly more interpersonal pressure than the low symptomatology group. Again such a distinction could not be made on the basis of a high or low score on the RS scale. The finding that low symptomatology women were similar in stability of pressure judgments to the pill women thus supported our conclusions that pill women were more consistent in their judgments of interpersonal pressure since they experienced attenuated mood fluctuations due to the reduced menstrual cycling associated with combination low-progestin oral contraceptives.

It is interesting to notice that although women were found to be less consistent than men on perceived pressure, no group of women was found to significantly exceed men on the absolute values of perceived pressure.
The relationships obtained in the present experiment, in which suspicion by subjects concerning any aspects of the major hypotheses was apparently nonexistent, and in which the menstrual cycle as a topic was in no way made salient, suggest physiological rather than socialcultural or attributional mechanisms underlying tension as here operationalized. However, as with any correlational design in which different existent groups are compared, alternate explanations can be formulated. The major alternative explanation that pill women may be more stable than non-pill women because they expect such effects from the pill doesn't seem warranted since Paige ${ }^{3}$ showed that a large majority of women have no such expectations. Yet, in no way do our statements signify that social and attributional processes do not play an important role in the variability of moods across the menstrual cycle.

The question of stability-instability of moods is an important one to establish in its own right since the variability in psychological experiments using female subjects could be due to some cyclical variation associated with the menstrual cycle. This point becomes especially crucial if menstruation were not evenly distributed over the calendar month. Although their data are far from conclusive, this contingency has been suggested by Malek et al. ${ }^{18}$ who have reported the lowest incidence of menstruation at new moon.

The authors are grateful to Tracy K. Luster for his help in producing videotaped vignettes, and to Kathy Orr for conducting part of the experimental sessions.

Requests for reprints should be sent to Paula Englander-Golden, Department of Human Relations, 601 Elm Street, Room 730, University of Oklahoma, Norman, Oklahoma 73019.

\section{INDEX TERMS}

menstruation, premenstrual tension, emotions, stress, contraceptives-oral.

\section{REFERENCES}

1. Moos, R. H. "The Development of a Menstrual Distress Questionnaire," Psychosom. Med., Vol. 30, 1968, pp. 853-867. 
2. Parlee, M. B. "The Premenstrual Syndrome," Psychol. Bull., Vol. 80, 1973, pp. 454-465

3. Paige, K. E. "Effects of Oral Contraceptives on Affective Fluctuations Associated with the Menstrual Cycle," Psychosom. Med., Vol. 33, 1971, pp. 515537.

4. Grant, E. C. G., and J. Pryse-Davies. "Effects of Oral Contraceptives on Depressive Mood Changes and on Endometrial Monoamine Oxidase and Phosphates," Br. Med. J., Vol. 3, 1968, pp. 777780.

5. Broverman, D. M., E. L. Klaiber, W. Vogel, and Y. Kobayashi. "Short-term versus Long-term Effects of Adrenal Hormones on Behaviors," Psychol. Bull., Vol. 81, 1974, pp. 672-694.

6. Briggs, M., and M. Briggs. "Relationship Between Monoamine Oxidase Activity and Sex Hormone Concentration in Human Blood Plasma." J. Reprod. Fertil., Vol. 29, 1972, pp. 447-450.

7. Klaiber, E. L., Y. Kobayashi, D. M. Broverman, and F. Hall. "Plasma Monoamine Oxidase Activity in Regularly Menstruating Women and in Amenorrheic Women Receiving Cyclic Treatment with Estrogens and a Progestin," J. Clin. Endocrinol. Metab., Vol. 33, 1971, pp. 630-638.

8. Koeske, R. K., and G. F. Koeske. "An Attributional Approach to Moods and the Menstrual Cycle," J. Pers. Soc. Psychol., Vol. 31, 1975, pp. 473-478.

9. Byrne, D. "The Repression-Sensitization Scale: Rationale, Reliability, and Validity," J. Pers., Vol. 29, 1961, pp. 344-349.
10. Lazarus, R. S., J. R. Averill, and E. M. Opton. "The Psychology of Coping: Issues of Research and Assessment," Coping and Adaptation, G. V. Coelho, D. A. Hamburg, and J. E. Adams, eds. Basic Books, New York, 1974.

11. Bardwick, J. M. Psychology of Women: A Study of Biocultural Conflicts. Harper \& Row, New York, 1971.

12. Ivey, M., and J. M. Bardwick. "Patterns of Affective Fluctuation in the Menstrual Cycle," Psychosom. Med., Vol. 30, 1968, pp. 336-345.

13. Page. M. "Postexperimental Assessment of Awareness in Attitude Conditioning," Ed. Psychol. Measurement, Vol. 31, 1971, pp. 891-906.

14. Williams, R. H. Textbook of Endocrinology. W. B. Saunders, Philadelphia, 1974.

15. Klaiber, E. L. Personal communication, 1976.

16. Edwards, A. L. Experimental Design in Psychological Research. Holt. Rinehart \& Winston, New York, 1950.

17. Byrne, D., J. Barry, and D. Nelson. "Relation of the Revised Repression-Sensitization Scale to Measures of Self-description," Psychol. Rep., Vol. 13, 1963, pp. 323-334.

18. Malek, J., J. Gleich, and V. Maley. "Characteristics of the Daily Rhythm of Menstruation and Labor," Ann. N. Y. Acad. Sci., Vol. 98, 1962. pp. 10421055. 